\title{
THE EFFECT OF TRAFFIC COMPLEXITY AND SPEED ON YOUNG AND ELDERLY PEDESTRIANS' STREET-CROSSING DECISIONS
}

\author{
Aurèlie Dommes ${ }^{1}$, Sabine Langevin ${ }^{1}$, Viola Cavallo ${ }^{1}$, Jennifer Oxley ${ }^{2}$, \& Fabrice Vienne ${ }^{3}$ \\ ${ }^{1}$ French Institute of Science and Technology for Transport, \\ Development and Networks (IFSTTAR - INRETS), LPC, Versailles, France \\ ${ }^{2}$ MUARC, Monash University Sunway Campus, Malaysia \\ ${ }^{3}$ French Institute of Science and Technology for Transport, \\ Development and Networks (IFSTTAR - INRETS), LEPSIS, Paris, France \\ Email: aurelie.dommes@ifsttar.fr
}

\begin{abstract}
Summary: This experiment aimed at studying the effects of age, traffic complexity and speed of the approaching cars on the probability of a pedestrian to be involved in a crash. Fifty nine participants aged between 20-84 years took part in a street-crossing estimation task in a simulated road environment. The results showed an overall higher number of 'collisions' with increasing age. While the number of collisions did not vary according to traffic complexity and speed of the approaching cars in the young group, the older participants were more likely to make decisions that led to collisions when the traffic was approaching from two rather than one direction, and at a high speed. The findings were discussed in relation to the effects of age-related cognitive and perceptual limitation on difficulties in selecting safe gaps. The present results have implications for improving older pedestrians' safety in terms of road design, speed reduction measures, and training opportunities.
\end{abstract}

\section{INTRODUCTION}

Elderly people are known to be over-involved in street-crossing fatalities. In France, more than half of all pedestrians killed on the road (51\%) are over 65 years of age, whereas this age group represents less than $15 \%$ of the population (ONISR, 2008). International crash data show the same trends. In spite of this obvious safety challenge, few studies have been dedicated to the safety of senior pedestrians. Research addressing street-crossing behavior has made progress only in the last 10-15 years. In addition to observational methods (e.g., Oxley et al., 1997), virtual environments have come into use and have significantly contributed to a better understanding of pedestrian behavior (e.g., Holland and Hill, 2010; Lobjois and Cavallo, 2007, 2009; Neider et al., 2010; Oxley et al., 2005).

Previous research (Holland and Hill, 2010; Lobjois and Cavallo, 2007, 2009; Oxley et al., 1997, 2005) pointed out several age-related changes in street-crossing behavior, such as slowing of decision-making, decreased walking speed, and difficulty in selecting safe gaps and adopting sufficient safety margins. The observational study of Oxley et al. (1997) showed that the increase of unsafe road crossing decisions in seniors appeared mostly in complex traffic situations such as two-way undivided roads, whereas their street-crossing safety significantly improved in less complex traffic such as one-way roads. Experimental studies also highlight age as a risk factor, together with the speed of the approaching cars (Lobjois and Cavallo, 2007, 2009; Oxley et al., 2005). Similarly to observational study findings, older adults were found to adopt shorter safety 
margins when the speed of the approaching vehicles was high, leading to significantly more unsafe decisions at higher speeds than lower speeds in simulated traffic environments.

The aim of the present experiment was to gain a better understanding of the risk factors that heighten the probability of older pedestrians to be involved in a crash. The present study is the first experiment that investigates the respective and combined effects of traffic complexity, speed and age in a safe and controlled environment. A simulated road-crossing environment was used, allowing a perfect control of the task constraints and graduated levels of task difficulty. Because of age-related cognitive and perceptual limitations, and accordingly to Oxley et al. (1997) findings, we hypothesized that older pedestrians would experience more difficulty than younger pedestrians in selecting safe gaps when traffic approached from two rather than one directions, as crossing two-lane roads involves assessing both directions simultaneously and making decisions based on both sources of traffic information. As older pedestrians tend to look first at the nearside and then at the far-side traffic prior to the road crossing (Geruschat et al., 2003), difficulties of older pedestrians in allocating attention to two simultaneous sources of information could be more marked if the oncoming traffic arrived at the pedestrians' position in the far lane first. This situation might lead the pedestrian to observe the far side before choosing a gap in the near side, thereby increasing the complexity of the task. As observed in previous studies (Lobjois and Cavallo, 2007, 2009), a high number of unsafe crossings with increasing speed should be also observed in older pedestrians, in one-way as well as in two-way traffic conditions.

\section{METHOD}

\section{Participants}

Fifty nine subjects participated in the experiment: 20 young ( 7 men and 13 women from $20-35$ years of age; mean 28.9; SD 4.21); 19 younger-old (8 men and 11 women from 60-67 years of age; mean 62.50; SD 2.41) and 20 older-old individuals (10 men and 10 women from 68-84 years of age; mean 76.55; SD4.44). The elderly participants underwent a medical examination to ensure the absence of severe physical or mental pathologies.

\section{Experimental Setup}

The street-crossing simulation device included an image-generation system, a rear-screen projection system, a 3D sound-rendition system and a recording system. The visual scenes were projected on six screens $(2.55 \mathrm{~m}$ high and $1.88 \mathrm{~m}$ wide) positioned in front of a standing pedestrian, and forming a semicircle around him/her (providing a horizontal visual field of $180^{\circ}$ and a vertical visual field of $40^{\circ}$ ). The pedestrian was positioned in such a way that he/she can turn his/her head to the left or right to watch the traffic coming from two directions. The simulated viewpoint corresponded to the edge of the curb where the participant was standing. The scene represented a two-way road 6 meters wide sidewalk-to-sidewalk. Traffic consisted of a series of motorcycles and cars approaching on the near-side lane, and/or on the far-side lane. On the near-side lane, vehicles approached from left to right with reference to the pedestrian standing at the sidewalk. Vehicles in the far lane were approaching from the right-hand side. 


\section{Experimental Task, Design and Procedure}

Participants were positioned at the edge of the simulated sidewalk facing the experimental road and looked left and right to the simulated road environment and approaching vehicles. They were instructed to choose gaps in the traffic in which they would have crossed and indicate when they would cross the entire road to the far sidewalk by pressing a response button. If they refused to cross, they waited for the next trial.

Traffic complexity was varied: flow(s) of vehicles (from 5 to 10 vehicles) could either approach on the near-side lane only, or from the two directions. In scenarios when traffic approached from both ways, the first vehicle of each traffic flow could reach the crossing line either in the near- or far-side lane first. Three conditions varying in traffic complexity were manipulated: (1) 'Singleway traffic approaching in the near lane only': the participant had to judge whether there was a sufficient time gap in the near-side approaching traffic to cross the entire two-way road; (2) 'Two-way traffic with vehicles reaching the pedestrian's position in the near lane first': the participant had to judge whether there was a sufficient time gap in the near-side approaching traffic to cross the first lane as well as a sufficient lap in the second lane before the arrival of the far-side traffic; (3) 'Two-way traffic with vehicles reaching the pedestrian's position in the far lane first': the pedestrian had to judge whether there were two sufficient time gaps simultaneously available in both lanes to cross the two-way traffic. In each traffic condition, vehicle speed (40 and $60 \mathrm{~km} / \mathrm{h}$ ) and time gap between target cars (or laps of arriving cars) of the traffic flow(s) (1 to $7 \mathrm{~s}$, in 1-s increments) were varied. The variable time gap appeared 3 seconds after the onset of the scene when traffic was approaching from one direction, and 6 seconds after scene onset when traffic was coming from both directions of the two-lane road. The time gap manipulation was intended to introduce random variation between trials. A 3 (age) $\mathrm{x} 3$ (condition) x 2 (speed) mixed design was used. A total of 162 trials was presented in a random order and divided into four blocks, with a break between blocks. The experiment started with some practice trials and lasted about one hour. Walking speed over a distance equivalent to the width of the simulated road (6 meters) was also measured in a corridor next to the simulator room (mean of three trials).

\section{RESULTS}

Several performance measures were considered for analysis including walking speed, decision delay, safety margin and gap accepted (see Table 1). Decision delay was calculated in reference to the start of the gap chosen by the participant in the near lane to cross the entire road: decision delay was equal to the time between the moment when the rear end of the first car of the gap chosen in the near lane passed in front of the pedestrian and the moment when the participant pressed the response button to declare the beginning of his/her crossing. Decision delay was negative when the participant decided to cross before the car passed the crossing line and positive after that. Safety margin referred to the time each individual would have taken to walk the distance of the virtual road (6 meters when traffic came from both directions and 3 meters when the one-way traffic approached in the near lane only) deducted from the gap remaining when he/she decided to cross. The gap accepted was the recorded as the time of an oncoming vehicle from the participant at the moment when he/she decided to cross. 
These measures were first input into one-way ANOVAs to examine the global effects of age. The results revealed a significant effect of age on walking speed $(\mathrm{F}(2,56)=11.08, \mathrm{p}<.0001$, $\left.\eta^{2}=.28\right)$, decision delay $\left(\mathrm{F}(2,56)=3.4, \mathrm{p}<.05, \eta^{2}=.11\right)$, and safety margin $(\mathrm{F}(2,56)=12.9, \mathrm{p}<.001$, $\left.\eta^{2}=.32\right)$ : young participants walked faster, decided to cross sooner when a gap was available, and therefore obtained larger safety margins than the two groups of old participants. Older-old participants showed very short safety margins, which suggests that they chose too short gaps to cross the street considering their mean walking speed and decision delay. Statistical analyses on gap accepted actually revealed a significant effect of age $\left(F(2,56)=4.8, p<.05, \eta^{2}=.15\right)$ : the average gap chosen by the older-old participants was significantly smaller than that selected by the younger-old participants $(\mathrm{p}<.01)$, and no significant difference appeared between older-old participants and young participants.

Table 1. General characteristics of the decisions made by the participants in the estimation task

\begin{tabular}{lccc}
\hline & Young & Younger-old & Older-old \\
\hline Walking speed (meter/seconds) & $1.23(0.13)$ & $1.12(0.24)$ & $0.96(0.16)$ \\
Decision delay (seconds) & $0.65(0.28)$ & $0.72(0.36)$ & $0.84(0.38)$ \\
Safety margins (seconds) & $1.61(0.59)$ & $1.25(1.19)$ & $0.28(0.99)$ \\
Gap accepted (seconds) & & & \\
$\quad$ Single-way traffic : accepted time gap in the near lane & $3.80(0.25)$ & $4.13(0.66)$ & $3.61(0.49)$ \\
$\quad$ Two-way traffic: accepted time gap in the near lane & $3.84(0.34)$ & $3.95(0.63)$ & $3.66(0.53)$ \\
$\quad$ Two-way traffic: accepted time gap in the far lane & $3.66(0.31)$ & $3.79(0.62)$ & $3.56(0.50)$ \\
\hline
\end{tabular}

*means and standard deviations in parentheses

These first results suggest that the older-old participants would have been at high risk when making such decisions in a real environment. Decisions that would have led to collisions with the approaching cars were therefore of particular interest to identify possible risk factors, such as age, traffic complexity and speed of the approaching cars. Virtual 'collisions' were calculated from the positions of the vehicles on the virtual road, the mean decision delay and walking speed of the participant. These data were expressed as a percentage of the number of accepted crossings by the participant.

The percentage of collisions was subjected to an age ${ }_{3} \mathrm{x}$ traffic complexity $\mathrm{x} \mathrm{speed}_{2}$ ANOVA. The analysis revealed a significant effect of age, $F(2,56)=16.4, p<.001, \eta^{2}=.37$. Post hoc tests indicated that older-old participants made significantly more decisions that led to collisions with the approaching cars (mean 35.5\%; SD 24.8) than young participants (mean 7.1\%; SD 8.9) and younger-old participants (mean 16.6\%; SD 20.3) $(\mathrm{p}<.001)$; the difference between young and younger-old participants was marginal $(\mathrm{p}=.08)$. The results also showed a significant effect of traffic complexity, $F(2,112)=7.6, p<.001, \eta^{2}=.12$. Post hoc analysis demonstrated a significantly higher percentage of collisions when the traffic coming from two directions was first arriving in the far lane (mean 23.4\%; SD 26.3) than in the near lane (mean 19.8\%; SD 23.9) or when the single-way traffic approached in the near lane only (mean $16.7 \%$; SD 15.2) $(\mathrm{p}<.05)$. Although the difference was marginal $(\mathrm{p}=.08)$, the two-way traffic arriving first in the near lane resulted in more collisions than the single-way traffic approaching only in the near lane. The effect of speed was also significant, $F(1,56)=22.2, p<.001, \eta^{2}=.28$. Cars approaching at $60 \mathrm{~km} / \mathrm{h}$ led to more collisions (mean 22.5\%; SD 21.1) than cars approaching at $40 \mathrm{~km} / \mathrm{h}$ (mean 17.5\%; SD 23.5). 
There was a significant interaction between age and traffic complexity, $F(4,112)=7.3, p<.001$, $\eta^{2}=.21$. While traffic complexity did not affect the percentage of collisions in young participants $(\mathrm{p}=.54)$, younger-old and older-old participants had significantly more collisions in the two conditions of two-way traffic compared with the single-way traffic environment $(p<.05)$. Older-old participants had significantly more collisions when the two-way traffic arrived in the far lane first than when it arrived in the near lane first $(p<.05)$. In contrast, this difference was not significant in the younger-old group of participants $(p=.36)$.

There was a significant age $\mathrm{x}$ speed interaction $\mathrm{F}(2,56)=5.2, \mathrm{p}<.01, \eta^{2}=.16$. Whereas collisions did not vary significantly as a function of speed in the young group (mean 6.8\%; SD 8.23 at 40 vs. mean $8.6 \%$; SD 9.5 at $60 \mathrm{~km} / \mathrm{h}, \mathrm{p}=.34$ ), older-old subjects made significantly more decisions that would have resulted in a collision at high speed (mean 40.2\%; SD 23.8) than at low speed (mean 30.7\%; SD 24.9) $(\mathrm{p}<.05)$. The speed difference fell short of statistical significance in the younger-old group (mean 14.9\%; SD 18.8 at 40 vs. mean 18.4\%; SD 21.8 at $60 \mathrm{~km} / \mathrm{h}, \mathrm{p}=.056$ ).

The traffic complexity $x$ speed interaction was also significant $F(2,112)=4.8, p<.05, \eta^{2}=.08$. The rise of collisions with increasing speed was significant only in the one-way traffic situation $(\mathrm{p}<.001)$. Collisions were just about as numerous in $40 \mathrm{~km} / \mathrm{h}$ conditions as at $60 \mathrm{~km} / \mathrm{h}$ in both two-way traffic situations: speed differences were marginal when the traffic coming from the two directions was arriving in the near lane first $(\mathrm{p}=.06)$ and non significant when the two-way traffic was arriving in the fare lane first $(\mathrm{p}=.09)$.

The age $x$ traffic complexity $x$ speed interaction was significant $F(4,112)=3.3, p<.05, \eta^{2}=.11$. Older-old participants made the highest number of decisions that led to collisions with the approaching cars when the traffic coming from the two directions was arriving at a high speed in the far lane first (45\%, see Figure 1). Indeed, post hoc tests showed that differences between the older-old and the two other groups were almost all significant, and particularly marked when the traffic was approaching at a high speed from one as well as two directions $(p<.05)$. The only non significant difference between groups concerned the low speed in the single-way traffic situation.

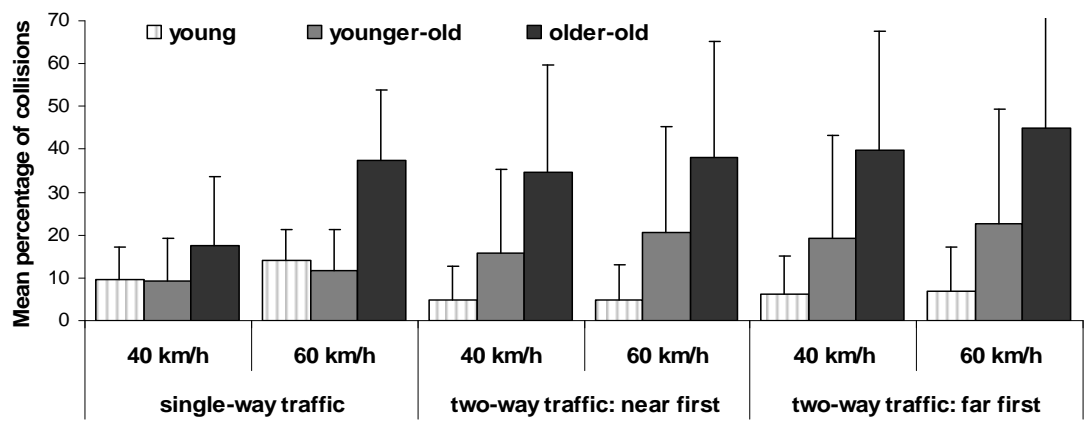

Figure 1. Mean percentage of collisions according to age, traffic complexity, and vehicle speed

\section{DISCUSSION}

The findings of this experiment showed age as an important risk factor for the safety of the crossing decisions made in a simulated pedestrian environment. Elderly participants were found to choose similar average time gaps as young adults did, although they walked slower and decided to cross later when a gap was available. These choices resulted in smaller safety margins 
and more virtual crashes: one out of three of their decisions led to virtual collisions compared to less than one collision out of ten decisions made by the young participants.

As suggested in the observational study of Oxley et al. (1997), complexity of the traffic environment appeared to be particularly detrimental for elderly pedestrians. While traffic complexity did not affect the number of decisions that led to collisions in the group of young adults, elderly participants experienced more virtual collisions when the traffic was approaching from two rather than one direction. Age-related limitations in allocating attention to two simultaneous sources of information may explain this finding.

Surprisingly, we also observed a quite high number of collisions $(16 \%)$ in the single-way traffic condition. Lower rates of collisions (near zero) have been observed in earlier studies (Lobjois and Cavallo, 2009) when a one-way oncoming traffic was approaching in a single lane. This difference may be explained by the fact that the present experiment as a whole was more complex than the previous studies insofar as the participants could not anticipate if the traffic was coming from one or two directions. The present findings may however be more relevant than past research and applicable to the real world situations where traffic is unpredictable. All trials were randomized in such a way that when the traffic approached in the near side, participants had to check if traffic was also approaching in the far-side lane of the road. Such a situation has not been tested in earlier studies, where traffic always arrived from one direction. Our results confirmed the increased difficulties with aging in interacting with complex traffic situations as noted in observational studies (Oxley et al., 1997). Older participants were found to be at a particularly high risk in the most complex condition of our experiment, i.e. when vehicles approached from both directions and the closest vehicle arrived in the far-side lane first. In this situation, pedestrians would be required to observe the far side of the road before choosing a gap to cross in the near-side lane. This strategy may be particularly unusual for older pedestrians who appear to make judgments with regard to the near-side traffic and begin to cross without consideration of the far-side traffic (Oxley et al., 1997).

Elderly pedestrians were also found to have difficulties to safely handle situations when vehicles approached at high speed. This finding is in line with earlier studies (Lobjois \& Cavallo, 2007; Oxley et al., 2005) indicating that seniors use simplified heuristics based on vehicle distance while neglecting speed information. For a given available time gap, the distance of the approaching car is necessarily greater at high speeds than at low speeds. Considering only the greater distance, the elderly more often decide that it is safe to cross when the speed of the approaching vehicles is high than when it is low (shorter distances). The use of distance-based heuristics is however dangerous when cars approach at high speeds because the time available for crossing is overestimated. High vehicle speed is undeniably a safety-critical factor for senior pedestrians. It not only affects decision-making and increases the probability of collisions, but also worsens its consequences for a population that is particularly vulnerable.

Overall, the findings of this study suggest that age-related cognitive declines may contribute to the difficulties in selecting safe gaps in the traffic in which to cross the road, especially in busy and high speed conditions and these difficulties may include impaired attention abilities and reduced executive functions (Salthouse et al., 2003). The decline in visual motion sensitivity (Snowden and Kavanagh, 2006) may also contribute to seniors ignoring the vehicles' visual movement and disregard speed information. 


\section{CONCLUSIONS}

This study suggests that traffic complexity and speed of approaching cars were important risk factors for the safety of elderly pedestrians. These factors seemed to interact, with a high risk of virtual crashes when traffic approached from two directions, when vehicles approached at high speed and when they arrived in the far lane first. These findings were observed in a simulated road environment which may not give a perfect assessment of crash frequency; they therefore deserve to be further investigated in real on-road tasks.

The results of this study however suggest several ways for improving elderly pedestrians' safety. Car-free islands in the middle of two-way roads could help the elderly to cross in two stages. This not only lowers their proneness to accidents by decreasing the time spent in the street, but also lightens the cognitive load of the street-crossing task. They also provide a refuge to pause safely in the middle of the road if needed. Speed reduction measures are clearly important for pedestrian safety and also recommended. Measures such as lowered speed limits as well as traffic calming techniques (e.g., road narrowing, pavement realignment, speed humps, etc) in areas of high older pedestrian activity should be considered. Furthermore, cognitive training programs can be a promising route worth exploring as a supplement to the above safety regulations and ergonomic measures.

\section{REFERENCES}

Geruschat, D.R, Hassan, S.E. \& Turano, K.A. (2003). Gaze behavior while crossing complex intersections. Optometry and Vision Science, 80(7), 515-528.

Holland C \& Hill R. (2010). Gender differences in factors predicting unsafe crossing decision in adult pedestrians across the lifespan: a simulation study. Accident Analysis \& Prevention, 42, 1097-1106.

Lobjois, R. \& Cavallo, V. (2007). Age-related differences in street-crossing decisions: The effects of vehicle speed and time constraints on gap selection in an estimation task. Accident Analysis \& Prevention, 39, 934-943.

Lobjois, R. \& Cavallo, V. (2009). The effects of aging on street-crossing behavior: from estimation to actual crossing. Accident Analysis \& Prevention, 41, 259-267.

Neider, M.B., McCarley, J.S., Crowell, J., Kaczmarski, H. \& Kramer, A.F. (2010). Pedestrians, vehicles, and cell phones. Accident Analysis and Prevention, 42, 589-594

Observatoire National Interministériel de Sécurité Routière (ONISR) (2008). Le bilan de l'accidentologie de l'année 2008. Paris: La Documentation Française.

Oxley, J.A., Fildes, B.N., Ihsen, E., Charlton, J.L. \& Day, R.H. (1997). Differences in traffic judgements between young and old adult pedestrians. Accident Analysis \& Prevention, 29, 839-847.

Oxley, J.A., Ihsen, E., Fildes, B.N., Charlton, J.L. \& Day, R.H. (2005). Crossing roads safely: An experimental study of age differences in gap selection by pedestrians. Accident Analysis \& Prevention, 37, 962-971. 
Salthouse T.A., Atkinson T.M. \& Berish D.E. (2003). Executive functioning as a potential mediator of age-related cognitive decline in normal adults. Journal of Experimental Psychology: General, 132, 566-594.

Snowden, R.J. \& Kavanagh, E. (2006). Motion perception in the aging visual system: minimum motion, motion coherence, and speed discrimination thresholds. Perception, 35, 9-24. 